\title{
Recent progress in distributed feedback InGaN/GaN laser diodes
}

Thomas J. Slight, Scott Watson, Shaun Viola, Amit Yadav, Szymon Stanczyk, et al.

Thomas J. Slight, Scott Watson, Shaun Viola, Amit Yadav, Szymon Stanczyk, Szymon Grzanka, Steffan Gwyn, Edik Rafailov, Piotr Perlin, Stephen P. Najda, Mike Leszczyński, Mohsin Haji, Anthony E. Kelly, "Recent progress in distributed feedback InGaN/GaN laser diodes," Proc. SPIE 10939, Novel InPlane Semiconductor Lasers XVIII, 109390I (1 March 2019); doi: 10.1117/12.2507630

SPIE. Event: SPIE OPTO, 2019, San Francisco, California, United States 


\title{
Recent progress in distributed feedback InGaN/GaN laser diodes
}

\author{
Thomas J Slight*a, Scott Watson ${ }^{\mathrm{b}}$, Shaun Viola ${ }^{\mathrm{b}}$, Amit Yadav ${ }^{\mathrm{c}}$, Szymon Stanczyk ${ }^{\mathrm{d}}$, Szymon \\ Grzanka $^{\mathrm{d}}$, Steffan Gwyn ${ }^{\mathrm{b}}$, Edik Rafailov ${ }^{\mathrm{c}}$, Piotr Perlin ${ }^{\mathrm{d}}$, Stephen P Najda ${ }^{\mathrm{d}}$, Mike Leszczyński ${ }^{\mathrm{d}}$, \\ Mohsin Haji ${ }^{\mathrm{e}}$, Anthony E Kelly \\ ${ }^{a}$ Compound Semiconductor Technologies Global Ltd, Hamilton, UK, G72 0BN; \\ ${ }^{\mathrm{b}}$ School of Engineering, University of Glasgow, Glasgow, UK, G12 8LT; \\ ${ }^{\mathrm{c}}$ Aston University, Birmingham, UK, B4 7ET ; ${ }^{\mathrm{d}}$ Topgan, Sokołowska, Warsaw, Poland; \\ ${ }^{\mathrm{e}}$ National Physical Laboratory, Teddington, UK, TW11 0LW.
}

\begin{abstract}
Laser diodes based on Gallium Nitride $(\mathrm{GaN})$ are useful devices in a wide range of applications including atomic spectroscopy, data storage and optical communications. To fully exploit some of these application areas there is a need for a GaN laser diode with high spectral purity, e.g. in atomic clocks, where a narrow linewidth blue laser source can be used to target the atomic cooling transition.

We report on the continuous wave, room temperature operation of a distributed feedback laser diode (DFB-LD) with high-order notched gratings. The design, fabrication and characterization of DFB devices based on the (Al,In)GaN material system is described. A single peak emission at $408.6 \mathrm{~nm}$ with an optical power of $20 \mathrm{~mW}$ at $225 \mathrm{~mA}$ and a side mode suppression ratio (SMSR) of $35 \mathrm{~dB}$ was achieved. Additionally, we demonstrate the use of a GaN DFB-LD as a transmitter in visible optical communications system. We also present results from a DFB-LD optimized for laser cooling of $\mathrm{Sr}+$.
\end{abstract}

Keywords: Semiconductor lasers, Distributed feedback laser diodes, GaN, Notched grating, Sidewall grating, Lateral grating, Optical communications, Laser cooling.

\section{INTRODUCTION}

Performance of InGaN/GaN DFB-LDs has lagged behind GaAs and InP based devices due to a smaller number of application areas to drive development. Of all GaN DFB-LDs reported to date the side mode suppression ratio (SMSR) has been low and CW operation has rarely been reported [1]. For certain key applications such as cold-atom based sensing systems [2], spectroscopy [3] \& optical communication systems [4], the lasers used must exhibit high SMSR, $\mathrm{CW}$ operation, and precise wavelength targeting.

Single wavelength lasers in the (Al,In)GaN material system have been realised in various ways including use of either buried [1] or surface gratings [5]. Buried gratings require complex overgrowth steps which have the potential to introduce growth defects while surface grating designs can compromise the quality of the p-type top contact. In our DFB laser implementation, gratings are formed along the sidewalls of a ridge waveguide laser diode [6][7][8]. It's one of the simplest ways (in terms of complexity of fabrication) to achieve single wavelength operation and has the advantage that the sidewall grating can be designed and implemented entirely post growth once the emission wavelength is known. Additionally, the coupling coefficient is determined mainly by the planar layout of the grating rather than the etch depth, thereby increasing design freedom.

*tslight@compoundsemi.co.uk

Novel In-Plane Semiconductor Lasers XVIII, edited by Alexey A. Belyanin,

Peter M. Smowton, Proc. of SPIE Vol. 10939, 109390I - (c) 2019 SPIE

CCC code: $0277-786 \mathrm{X} / 19 / \$ 18 \cdot$ doi: $10.1117 / 12.2507630$ 


\section{DESIGN}

In this work we investigate a 39th order grating which has the benefit of easier fabrication with larger feature sizes over lower order gratings. The principal of operation for this device is similar to that described in [9], where essentially a Fabry-Perot (FP) laser is provided with weak optical feedback by a high order grating. The reflectivity provided from a 39 th order grating is substantially weaker than achieved in low order gratings and in this case the dominant source of optical feedback is from the cleaved facets. The effect of the grating is to introduce a wavelength selective loss for FP cavity modes away from the Bragg wavelength and hence lasing is in the single or narrow band of FP modes enveloped by the reflection bandwidth of the grating.

There are two main differences in how we have implemented this concept in a GaN laser compared to the equivalent device in InP. Firstly, the index perturbations are achieved using a sidewall grating rather than a surface grating. Since the entire top surface of the waveguide is electrically contacted, we avoid large optical losses in un-pumped regions.

The second difference in implementation is due the fact that the free spectral range of the Fabry-Perot cavity in a GaN laser is far smaller than for an InP based laser (e.g. for a $1000 \mu \mathrm{m}$ long resonator the FSR is $0.03 \mathrm{~nm}$ at $420 \mathrm{~nm}$ vs $0.4 \mathrm{~nm}$ at $1550 \mathrm{~nm}$ ) hence the reflection bandwidth of the grating must be much narrower for GaN. The grating bandwidth is dependent on the reflectivity of each notch pair; the lower the reflectivity per notch pair the narrower the bandwidth, but a larger number of pairs are required to maintain the required total reflectivity. We used the TMM (transmission matrix method) to estimate the number of notch pairs required. Effective modal indices were calculated using a 2D optical mode solver for both perturbed (notched) and unperturbed sections of the waveguide. The indices were then used along with a TMM solver to calculate the reflection bandwidth of the grating. This technique is approximate but gives us an estimate of the required quantity of notches to achieve single wavelength operation. Our design used a 39th order grating with 125 notch pairs along the ridge which we estimate to have a bandwidth $\sim 0.1 \mathrm{~nm}$.

\section{FABRICATION}

Devices were fabricated from AlInGaN laser epi-structures, consisted of three InGaN quantum wells sandwiched between $\mathrm{GaN}$ barriers, $\mathrm{GaN}$ waveguide layers and $\mathrm{Al}_{0.06} \mathrm{Ga}_{0.94} \mathrm{~N}$ cladding layers. The grating pattern was first written by ebeam lithography into ZEP 520 resist which was then transferred into a $120 \mathrm{~nm}$ thick layer of $\mathrm{SiO} 2$ using reactive ion etching (RIE). The ZEP resist was then removed to leave the $\mathrm{SiO}_{2}$ as a mask suitable for Inductively coupled plasma (ICP) etching. An optimized ICP process with a $\mathrm{Cl}_{2} / \mathrm{N}_{2}$ chemistry gave a smooth and vertical etch profile, important in achieving optimal grating performance. The as etched waveguide is shown if figure 1. Once etched, a layer of $\mathrm{SiO}_{2}$ was deposited to act as an insulator between the metal contact pads and the semiconductor. Via's were etched in this layer to contact the top of the waveguide ridge and a layer of $\mathrm{Ni} / \mathrm{Au}$ was deposited by evaporation to act as an ohmic contact. The sample was then thinned/polished, back metal deposited, and then cleaved into individual chips.

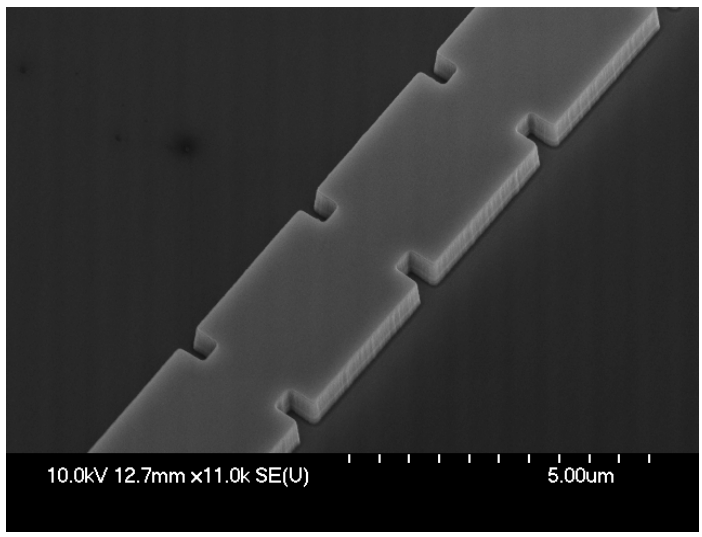

Figure 1. SEM image of the as etched $39^{\text {th }}$ order grating/waveguide. 


\section{CHARACTERISATION}

The samples were placed on a thermoelectric cooler (TEC) and stabilized to $20^{\circ} \mathrm{C}$. The laser diodes were driven by laser diode controller in $\mathrm{CW}$ operation. The optical power was measured using a standard photodiode power sensor connected to a power meter. The emission spectra were acquired using a high-resolution $1 \mathrm{~m}$ long spectrometer with a resolution of approximately $6.5 \mathrm{pm}$.

Figure 2a shows the voltage and optical power as a function of drive current for a 39th order grating DFB LD with 1.5 $\mu \mathrm{m} / 2.5 \mu \mathrm{m}$ grating widths, a cavity length of $1000 \mu \mathrm{m}$ and an etch depth of $520 \mathrm{~nm}$. The measured threshold current is $130 \mathrm{~mA}$ and the slope efficiency is $0.27 \mathrm{~W} / \mathrm{A}$ (for an uncoated device). The optical power characteristic displayed a kink at $160 \mathrm{~mA}$ which is thought to be due to mode hops between adjacent FP modes caused by differences in the shift of gain curve and RI with temperature. Figure $2 \mathrm{~b}$ shows the emission spectrum measured at a drive current of $225 \mathrm{~mA}\left(1.73^{*}\right.$ Ith), the SMSR is $35 \mathrm{~dB}$ at a CW optical output power of $20 \mathrm{~mW}$.

The notched lasers showed both two times higher threshold current and two times lower efficiency than Fabry-Perot lasers processed from the same wafer. We believe the compromised performance of the DFB-LD over the FP-LD is due to optical losses occurring in the notched section of the waveguide. By minimizing the number notch pairs while maintaining the bandwidth necessary for single wavelength operation it should be possible to improve performance. The application of HR/AR optical coatings to the laser facets should also reduce threshold current and increase slope efficiency.



Figure 2a. Optical power and voltage as a function of current.

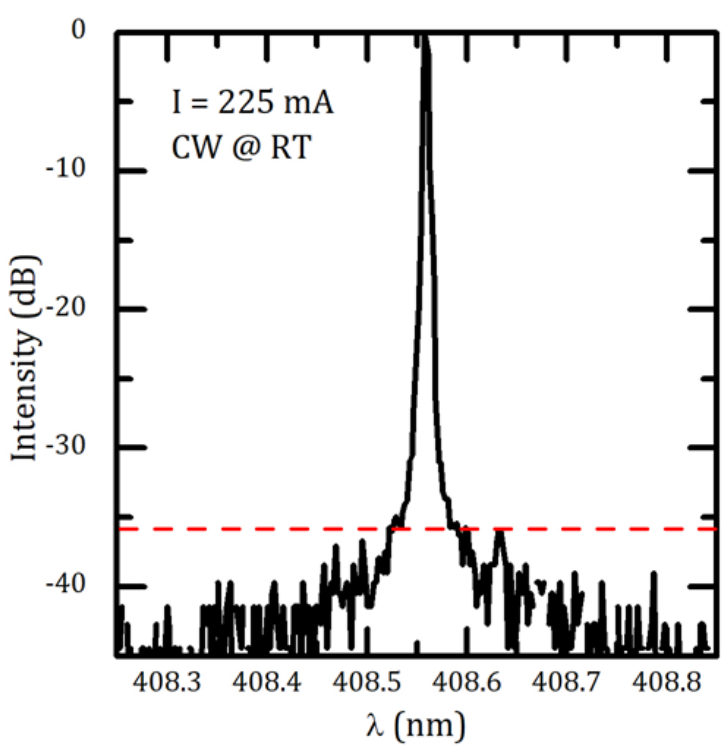

Figure 2b. Emission spectrum at a current of $225 \mathrm{~mA}$. 


\section{LASER COOLING}

The availability of suitable compact laser technology is key to the commercial development of cold-atom quantum enabled devices, such as atomic clocks, gravity sensors, accelerometers and gyroscopes. Popular divalent cold atom based systems require cooling at blue wavelengths $(397-461 \mathrm{~nm})$ which are typically reached by frequency doubling near-infrared sources; either using an extended cavity diode laser (ECDL) or Titanium Sapphire based arrangement feeding a temperature stabilised non-linear crystal. These systems require a quiet mechanical and thermal environment, are power hungry, costly to build, and prone to failure when used outside of the laboratory.

Robust, single mode blue laser diode sources based on the GaN/AlGaN material system offer a way forward without the complexity and bulk of frequency doubled systems. To this end we have developed a monolithic DFB laser diode to target the ${ }^{88} \mathrm{Sr}+$ cooling line at $422 \mathrm{~nm}$. Sr+ is widely considered to be one of the simplest ion systems available, primarily due to the simplicity in preparation of the quantum state for clock interrogation. This gives Sr+ clocks the potential to become much more compact than other optical atomic clock systems.

The DFB-LDs are similar to those described in the previous sections and were based on $750 \mu \mathrm{m}$ long waveguides with $39^{\text {th }}$ order notched gratings with a Bragg wavelength of $422 \mathrm{~nm}$. High reflectivity facet coatings were used to reduce the threshold current and compensate for scattering losses associated with the grating. An emission spectrum measured at a drive current of $110 \mathrm{~mA}$ is given in figure 3 .

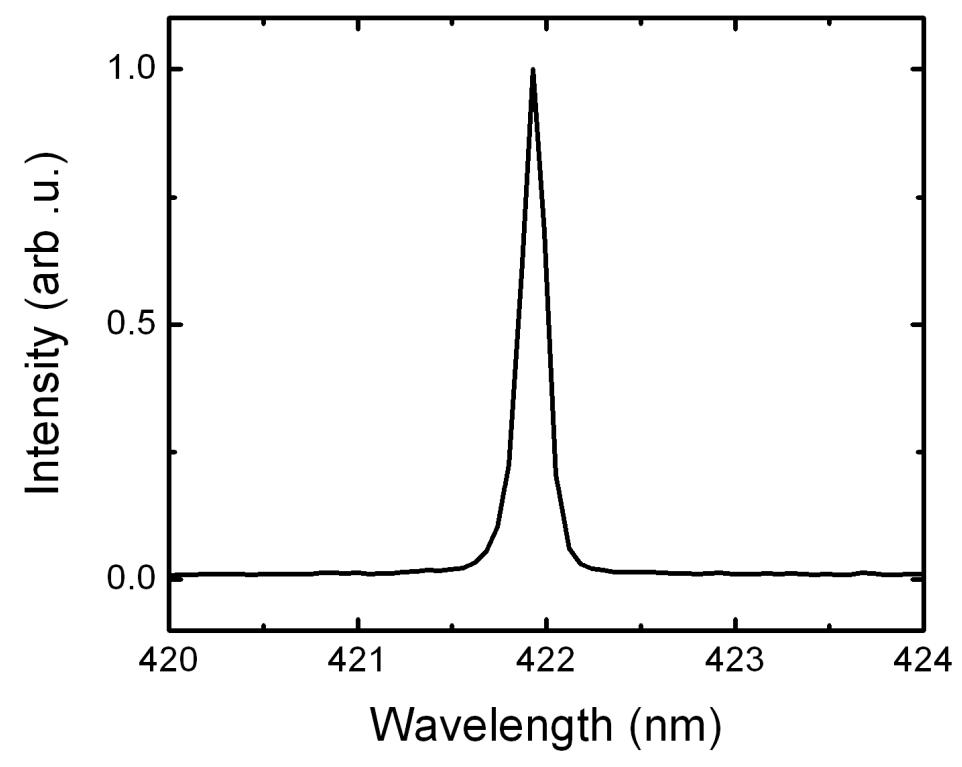

Figure 3. Emission spectra at drive current of $110 \mathrm{~mA}$.

\section{VISIBLE LIGHT COMMUNICATION}

In this section we describe experiments to evaluate GaN DFB-LDs for suitability as a transmission source in a visible light communications system. The experimental setup was as follows: The laser assembly was attached to thermoelectric cooler and operated at a fixed temperature of $25^{\circ} \mathrm{C}$. The laser diode output was collected and collimated with a one inch lens before being transmitted over a free space link of $10 \mathrm{~cm}$. It was then focused onto a Newport 818-BB-21A photoreceiver with a second one inch lens. The small signal frequency response was measured using a HP8753ES network analyser. The system frequency response was measured at drive currents from $185 \mathrm{~mA}$ to $230 \mathrm{~mA}$. The maximum optical bandwidth of the system was found at a drive current of $220 \mathrm{~mA}$ as shown in figure 5 . 


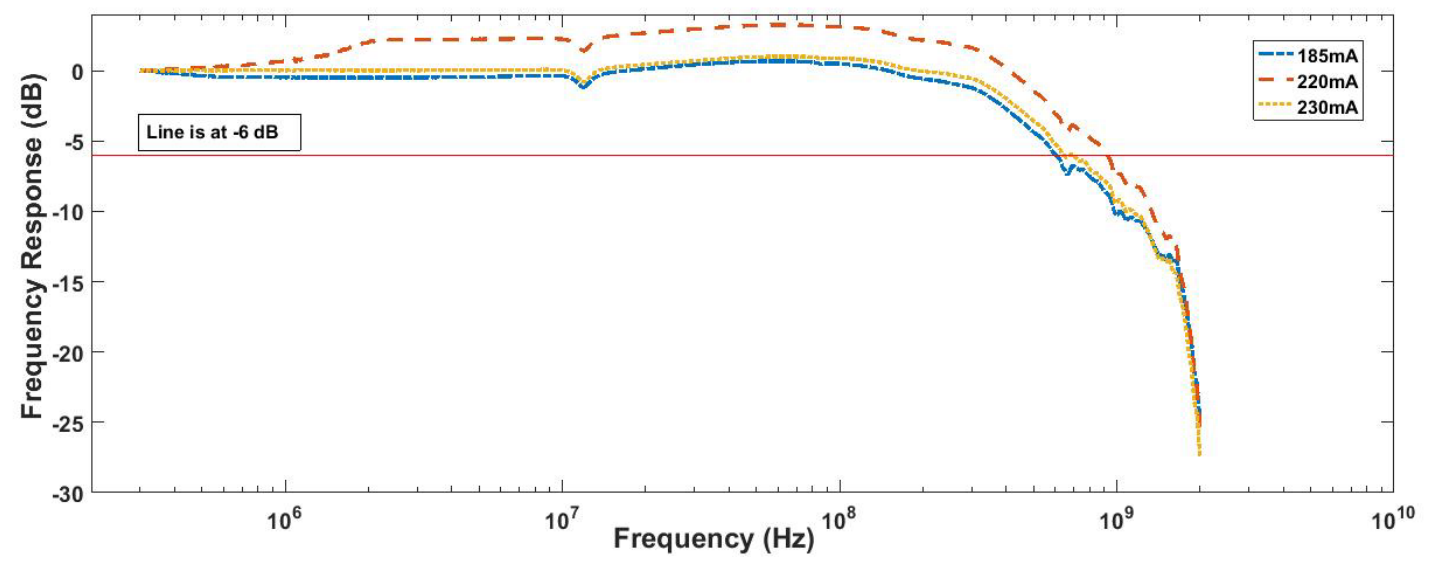

Figure 5. Small signal frequency response.

We also drove the laser using a NRZ-OOK modulation format. Eye diagrams were measured using an oscilloscope with a data rate of $1 \mathrm{Gbit} / \mathrm{s}$ as shown in figure 6. Bit error rate (BER) measurements of the NRZ-OOK system were measured using a BER detector. The laser was found to be capable of transmitting data error free up to $1.7 \mathrm{Gbit} / \mathrm{s}$.

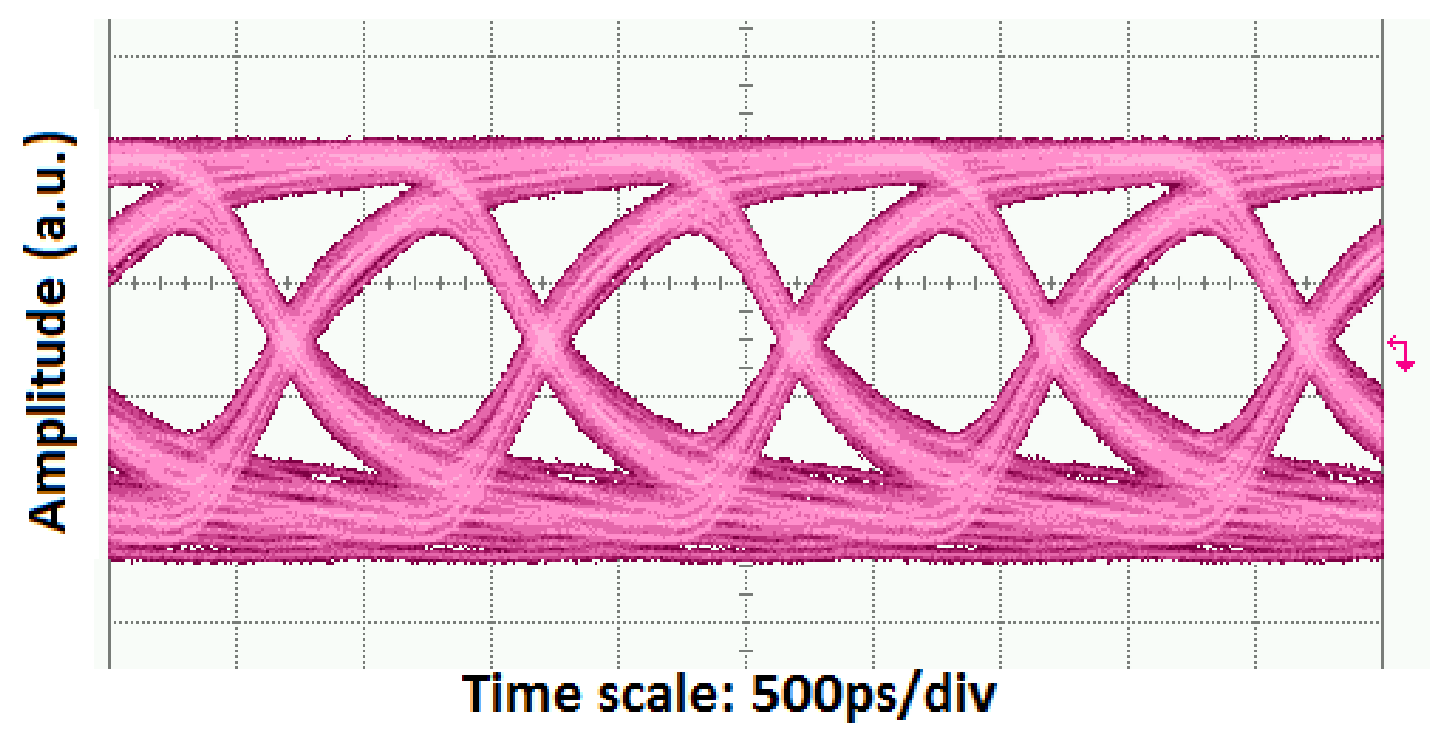

Fig.6. Eye diagram at $1 \mathrm{Gbit} / \mathrm{s}$ modulation rate.

\section{CONCLUSIONS}

We have demonstrated (Al,In)GaN DFB-LDs with CW single mode output powers of $20 \mathrm{~mW}$ and an SMSR better than $35 \mathrm{~dB}$. The design has a relatively simple fabrication route without regrowth and with more easily achievable feature sizes over lower order gratings. To further improve performance, optimised grating designs and anti-reflective facet 
coatings can be used in order to increase power and decrease threshold current. We have also demonstrated the application of a GaN DFB-LD as a transmitter in an optical communications system and show bit free data transmission at $1.7 \mathrm{Gbit} / \mathrm{s}$. We also presented results from a DFB-LD optimized for laser cooling of Sr+.

Acknowledgements: This research has been supported by the European Union with grant E10509, Innovate UK through grant 132543, and by the National Centre for Research and Development (E10509/29/NCBR/2017 and 1/POLBER3/2018 and project NCN-2013/11/B/ST3/04263).

\section{REFERENCES}

[1] S. Masui, K. Tsukayama, T. Yanamoto, T. Kozaki, S. I. Nagahama, and T. Mukai, "CW operation of the firstorder AlInGaN $405 \mathrm{~nm}$ distributed feedback laser diodes," Japanese J. Appl. Physics, Part 2 Lett., vol. 45, no. 46, pp. 1223-1225, 2006.

[2] A. Shiner, "Development of a frequency stabilized 422-nm diode laser system and its application to a $88 \mathrm{Sr}+$ single ion optical frequency standard," no. December, 2006.

[3] H. Leinen, D. Glässner, H. Metcalf, R. Wynands, D. Haubrich, and D. Meschede, "GaN blue diode lasers: a spectroscopist's view," Appl. Phys. B, vol. 70, no. 4, pp. 567-571, 2000.

[4] T.-C. Wu, Y.-C. Chi, H.-Y. Wang, C.-T. Tsai, and G.-R. Lin, "Blue Laser Diode Enables Underwater Communication at 12.4 Gbps," Sci. Rep., vol. 7, p. 40480, Jan. 2017.

[5] D. C. Sanz, J. M. Rorison, and S. Yu, "InGaN/GaN MQW Laser Diodes with 4th Order FIB-etched Gratings," in Quantum Electronics and Laser Science Conference, 2005, p. JTuC82.

[6] T. J. Slight, S. Stanczyk, S. Watson, A. Yadav, S. Grzanka, E. Rafailov, P.Perlin, S. P. Najda, M. Leszczyński, S. Gwyn, and A. E. Kelly, " Continuous-wave operation of (A1,In)GaN distributed-feedback laser diodes with high-order notched gratings," App. Phys. Exp, vol. 11, p. 112701, 2018.

[7] T. J. Slight, O. Odedina, W. Meredith, K. E. Docherty, and A. E. Kelly, "InGaN/GaN Distributed Feedback Laser Diodes With Deeply Etched Sidewall Gratings," IEEE Photonics Technol. Lett., vol. 28, no. 24, pp. 28862888, Dec. 2016.

[8] L. Hou, M. Haji, I. Eddie, H. Zhu, and J. H. Marsh, "Laterally coupled dual-grating distributed feedback lasers for generating mode-beat terahertz signals," Opt. Lett., vol. 40, no. 2, p. 182, 2015.

[9] R. Phelan, W. H. Guo, Q. Lu, D. Byrne, B. Roycroft, P. Lambkin, B. Corbett, F. Smyth, L. P. Barry, B. Kelly, J. O'Gorman, and J. F. Donegan, "A novel two-section tunable discrete mode Fabry-Pérot laser exhibiting nanosecond wavelength switching," IEEE J. Quantum Electron., vol. 44, no. 4, pp. 331-337, 2008. 